4. Without expressing an opinion in regard to the therapeutic value of the serum in the treatment of pneamonia, the test by
titrage in the mouse, when controlled by a standard serum, affords a basis for the international standardization of the serum. Therapeutic serum may be titrated in mice either by injecting intrapeutic serum may be titrated in mice either by injecting intraperitoneally a fixed quantity $(0.2$ c.cm.) of serum mixed with a highly virulent broth culture in doses ranging from $0.1 \mathrm{c.cm}$. to $0.4 \mathrm{c.cm}$. or more; or by injecting smaller and varying quantities $(0.001$ or $0.0001 \mathrm{c.cm}$.) of serum intraperitoneally, and three hours later a moderate dose of culture-for example, $0.0001 \mathrm{c.cm}$. The virulence of the culture should be such as to kill mice in doses of at least 0.0000001 c.cm. within forty eight hours. For every titration a standard serum must be used.

5. Further research should be made into the mechanism of recovery from pneumonia and into the mode of action of antipneumococcus serum.

6. A study of antistreptococcus serum is desirable.

7. Standard cultures agglutinating and standard therspeutic serums are to be supplied by the Central Institute of Copenhagen.

Dysentery.

The following resolutions were adopted

1. It is necessary to continue the experiments in order to determine the choice of the animal to be used for standardization of antidysentery serum. These tests should be carried out comparatively on rabbits weighing 1,500 to 1,600 grams and on white mice weighing 15 to 16 grams.

2. The substances to be compared are liquid toxin, solid toxin (Wellcome), and microbes killed by hest. (a) Liquid toxin will be prepared by each laboratory with culture toxic Shige which will be supplied by the Pasteur Institnte -namely the filtrate of a twenty days' old culture ou peptone broth (Chopton broth (Chapoptant's peptone) grown in litre flasks containin 500 c.cm. of liquid with an alkalinity PH 7.4. (b) The solid English toxin will be supplied by the Wellcome Laboratories. (c) The microbes killed by heating at $56^{\circ}$ for one hour and dried in vacunm will be supplied by the Pasteur Institate (aga culture emulsified in physiological salt solution). The effect of subcutaneous, intraperitoneal, and intravenous injections will be compared.

3. It is not necessary in making the tests to discriminate between the anti-neurctoxic and the anti-enterotoxic pcwer of the serum.

4. The titration of serums will be carried out by a mixture of toxin and serum, after thirty minutes' contact at laboratory temperature.

5 . It is not necessary to seek to give the serum an antiinfectious power as distinct from its antitoxic power.

6 . It would be of interest to test on man the power of monovalent serums (anti-Flexner, anti-His, etc.) against homologous strains of dysenteric organisms.

\section{NOTES ON SMALL-POX.}

$$
\text { BY }
$$

\section{W. MCCONNEL WANKLYN, B.A.Cantab.,} M.R.C.S., L.R.C.P., D.P.H.

\section{Outlook.}

At the time of writing (December 8th) small-pox in London appears, generally speaking, to be checked. The total cases to date are 62 with 20 deaths. No further cases have been reported from Lambeth, Bermondsey, or Poplar, and the rest of Loudon is apparently free.

There is thus a welcome lall, due mainly to effective medical team work in countering the disease, so that it finds more difficulty in gaining headway than formerly was the case. That is the true explanation of the phenomenon which at first may seem puzzling-namely, that small-pox appears not to be able to "get going" in London; and it is also the reason why no cases were reported during the last fortnight in spite of the previous sharp outburst. It is not the case that small-pox is a feeble thing which dies out of its own accord; the fact is that at present it is a formidable menace; but so far the counter-work las been too good for it. Only so far - the effort needs to be kept ap, if only because of the dis quieting fact that five of the recent foci have their origins unaccounted for; and the infection which started them may have started other foci still latent and shortly to burst out. Danger, therefore, is far from being past.

\section{The Poplar Outbreak.}

The suggestion that the recent Poplar outbreak consisted of a few dropping cases or an insignificant group in an area remote from and unconcerned with the rest of London, and that therefore the whole occurrence was trivial, is not in accordance with the facts. It is correct to say that there was a compact institution of about 1,000 souls, in which nearly all concerned were in the freest communication with the surrounding neighbourhood and were susceptible to small-pox; the institution actually overlooks the docks the part of Poplar in which it is situated being essentially a seaport town. In surveying the position beforehand this was regarded as a notable danger-spot for the rest of London, and in the event of an outbreal there was strong reason to expect very little assistance from vaccination, owing to the fact that the local authorities and many of the inhabitants were strongly opposed to that prophylactic. The general position may be gauged from the fact that in respect of about 4,500 children born in that borough in 1921 returns of successful vaccination had been received for about 1,000 only. Such were the admitted facts of the position.

This being the case, in this institution on October 13th a man fell ill of small-pox; he had the rash on October 15th ; it was not recognized. This was the earliest case that eventually could be heard of ; it is possible there was in the vicinity of the institution another undetected case with whom some of the inmates came in contact on and after October 10th. Be that as it may, till then the institution had a clean bill as regards small-pox. The abovementioned man moved freely about the institution between October 15th aud 28th, with such effect that, so far from the infection being the feeble thing which it has been represented to be, daring the week-end October 28 th to 30 th the institution and immediate neighbourhood was found to be variolously ablaze,
with 25 cases of small-pox.

London and its neighbourhood were most gravely menaced, if only from the fact that there had been discharged from the institution, since the first case had begun to infect those around him, a large number of the inmates; and from the fact that the various contacts comprised the following different classes of people:

(1) Other inmates remsining in the institation; (2) recently discharged inmates; (3) visitors to the inmates; (4) persons outside who had been visited by them; (5) medical and nursing staff. (6) scrubbers and other attendants ; (7) porters and other male ward staff; (8) occasional visitors to the wards, such as guardians and chaplains; (9) other staff, such as engineers, laundry hands, and others. Many of these persons had their 1 omes within a mile or two of the institution, and there was the best reason to consider the whole of that area as potentially dangerons.

When the first five cases were discovered, on tho morning of Saturday, October 28th, and it suddenly became apparent that the institution was already plentifully sown with infection; when all preventive measures had suddenly to be planned and started between 11 a.m. and 12.30 p.m. on a Saturday, when normal departmental activity ceases till the following Monday, though fire and small-pox do not: it is in strict accord with the fact to say that London at that moment was threatened with a catastrophe of appalling and almost unexampled gravity, likely to rival some of the worst epidemics of the past in expenditure of human life and treasure.

No one who was present is likely to forget the scene of consternation when the actual position was realized; nor the manner in which the emergency was faced and tackled, until in the end, after twenty-four hours of intensely strenuous work, it was completely mastered; so that then it was definitely said that, in spite of a score of cases scattered throughout that crowded and susceptible community, and in spite of large numbers of that community having been intimately exposed to infection, no further cases would occur in the institution after Wednesday, November 8th-that is, the short period of eleven days after the first discovery of the small-pox cases. Such in fact was the actual result, due of course to the preventive measures which had been taken. These were effected with remarkable promptitude-a triumph of combined team work on the part of all concerned; of which, while everyone did his utmost and no one seemed to lead, it should be said that the actual orders were given by the chairman of the House Committee, who was constantly on the spot-an English working man, formerly a stevedore, whose sagacity and courage, guided and fortified by medical advice and support, were primarily responsible for retrieving a desperate situation. Formerly a convinced antivaccinator, he now took fairly into account phenomena of which he had lad no previous experience; he witnessed the condition of the small-pox patients both at the institution and at the Small-pox Hospital, where he specially visited them; as a result he himself was vaccinated and influenced many others to adopt the same remedy. Like other antivaccinators whom the true facts have never reached, he honestly revised his attitude in regard to this question as soon as the facts came home to him.

These are some of the reasons why, to the surprise of many good judges, the Poplar outbreak "fizzled out." Why was it possible to achieve this result? The answer is, because the position had been well studied beforehand; because the previous quiet period had been fully and uncersingly occupied with advance preparation to meet any such emergency as did in fact arise. When, therefore, the crisis came, it was possible promptly and decisively to meet it. 